\title{
Application of Finite Element Method in High Frequency Simulations
}

\author{
G. Friedl \\ Széchenyi István University \\ 9026, Győr, Hungary \\ E-mail: friedl@maxwell.sze.hu
}

Abstract: This paper is written in the frame of the usage of finite element method at high frequencies. Simulations in time and frequency domain have their own methodology, for example the setting of the excitations and boundary conditions. The paper presents the weak formulations of both domains derived from Maxwell's equations and from the Sommerfeld radiation condition, simulations in isotropic and linear media, and if it is possible, comparisons between the simulations and analytical results. The first part of the paper is about the theory of the electromagnetics, the form of the wave equations in time and frequency domain, and about the nodal finite element method. The second chapter is about deriving the weak forms, and about their implementation method. The final chapter shows the implementation of the finite element method, and some results simulated in time and frequency domain with different geometry and boundary conditions.

Keywords: nodal finite element method, radio frequency simulation, time domain, frequency domain, Newmark method

\section{Introduction}

\subsection{Electromagnetics at high frequencies}

The basics of the theory of electromagnetism are defined by Maxwell's equations [1], that describe the behavior of the electromagnetic field in every moment and at every point in space for all frequencies. The equations have a form in time and frequency domain as well. The differential forms of Maxwell's equations in time domain for linear media [2-7] are the following:

$$
\begin{gathered}
\nabla \times \boldsymbol{H}(\boldsymbol{r}, t)=\boldsymbol{J}(\boldsymbol{r}, t)+\sigma \boldsymbol{E}(\boldsymbol{r}, t)+\varepsilon \frac{\partial \boldsymbol{E}(\boldsymbol{r}, t)}{\partial t} \\
\nabla \times \boldsymbol{E}(\boldsymbol{r}, t)=-\mu \frac{\partial \boldsymbol{H}(\boldsymbol{r}, t)}{\partial t},
\end{gathered}
$$




$$
\begin{aligned}
& \nabla \cdot \boldsymbol{B}(\boldsymbol{r}, t)=0, \\
& \nabla \cdot \boldsymbol{D}(\boldsymbol{r}, t)=\rho .
\end{aligned}
$$

Here, the field quantities are depending on space $\boldsymbol{r}$ and time $t \boldsymbol{H}=\boldsymbol{H}(\boldsymbol{r}, t)\left[\frac{\mathrm{A}}{\mathrm{m}}\right]$ indicates the magnetic field intensity, $\boldsymbol{J}=\boldsymbol{J}(\boldsymbol{r}, t)\left[\frac{\mathrm{A}}{\mathrm{m}^{2}}\right]$ is the current density, $\boldsymbol{D}=\boldsymbol{D}(\boldsymbol{r}, t)\left[\frac{\mathrm{C}}{\mathrm{m}^{2}}\right]$ is the electric displacement vector, $\boldsymbol{E}=\boldsymbol{E}(\boldsymbol{r}, t)\left[\frac{\mathrm{V}}{\mathrm{m}}\right]$ is the electric field intensity, $\boldsymbol{B}=$ $\boldsymbol{B}(\boldsymbol{r}, t)[\mathrm{T}]$ indicates the magnetic flux density and $\rho\left[\frac{\mathrm{C}}{\mathrm{m}^{3}}\right]$ is the charge density, the permittivity of vacuum is $\varepsilon_{0}=8.854 \cdot 10^{-12}\left[\frac{\mathrm{F}}{\mathrm{m}}\right]$, and the permeability of vacuum is $\mu_{0}=4 \pi \cdot 10^{-7}\left[\frac{\mathrm{H}}{\mathrm{m}}\right]$. The constitution relations in linear media have the form:

$$
\begin{gathered}
\boldsymbol{D}=\varepsilon_{0} \varepsilon_{r} \boldsymbol{E}, \\
\boldsymbol{B}=\mu_{0} \mu_{r} \boldsymbol{H}, \\
\boldsymbol{J}=\sigma\left(\boldsymbol{E}+\boldsymbol{E}_{i}\right) .
\end{gathered}
$$

Here, $\boldsymbol{E}_{i}$ denotes the so called impressed electric field. The first Maxwell's equation in (1) and the second one in (2) in the frequency domain under the condition $\sigma \ll \varepsilon \omega$ can be written [2-6] as:

$$
\begin{gathered}
\nabla \times \boldsymbol{H}(\boldsymbol{r}, \omega)=\boldsymbol{J}(\boldsymbol{r}, \omega)+\sigma \boldsymbol{E}(\boldsymbol{r}, \omega)+\mathrm{j} \omega \varepsilon_{0} \varepsilon_{r} \boldsymbol{E}(\boldsymbol{r}, \omega), \\
\nabla \times \boldsymbol{E}(\boldsymbol{r}, \omega)=-\mathrm{j} \omega \mu_{0} \mu_{r} \boldsymbol{H}(\boldsymbol{r}, \omega),
\end{gathered}
$$

where $\omega$ is the angular frequency and $\mathrm{j}$ is the imaginary unit. If there are no surface currents, on the boundary of the two media, the tangential component of the electric and the magnetic field intensity are continuous:

$$
\begin{aligned}
& \boldsymbol{n} \times\left(\boldsymbol{E}_{2}-\boldsymbol{E}_{1}\right)=0, \\
& \boldsymbol{n} \times\left(\boldsymbol{H}_{2}-\boldsymbol{H}_{1}\right)=0 .
\end{aligned}
$$

Above, $\boldsymbol{n}$ denotes the normal vector of the surface. The electric field is perpendicular to any perfectly conducting plane. At infinity, the electric field and the magnetic field must vanish, the electric field and magnetic field satisfies the Sommerfeld radiation condition [8]:

$$
\lim _{r \rightarrow \infty} r\left[\nabla \times \boldsymbol{E}+\mathrm{j} k_{0} \boldsymbol{n} \times \boldsymbol{E}\right]=\overrightarrow{0},
$$

where $r$ is the distance from the source of the electromagnetic field, and $k_{0}$ is the wave number in free space. The wave number is defined as:

$$
k_{0}=\omega \sqrt{\mu_{0} \varepsilon_{0}} .
$$




\subsubsection{Wave equation in time domain}

The wave equation for the electric field can be derived from (1) and (2) in time domain $[5,8]$ by eliminating the magnetic field intensity as:

$$
\varepsilon \frac{\partial^{2} \boldsymbol{E}}{\partial t^{2}}+\sigma \frac{\partial \boldsymbol{E}}{\partial t}+\nabla \times\left(\frac{1}{\mu} \nabla \times \boldsymbol{E}\right)=-\frac{\partial \boldsymbol{J}}{\partial t} .
$$

Simulations are performed in a finite space, the boundaries are not at the infinity, i.e. the Sommerfeld radiation condition is not usable in the form of (12). On the surface of a finite space, the radiation condition in time domain [8] can be approximated as:

$$
\boldsymbol{n} \times\left(\frac{1}{\mu_{0}} \nabla \times \boldsymbol{E}\right)+Y_{0} \boldsymbol{n} \times\left(\boldsymbol{n} \times \frac{\partial \boldsymbol{E}}{\partial t}\right) \approx 0,
$$

where $Y_{0}$ is the wave admittance of free space, and

$$
Y_{0}=\sqrt{\frac{\varepsilon_{0}}{\mu_{0}}}=\frac{1}{120 \pi}[S] .
$$

\subsubsection{Wave equation in frequency domain}

The wave equation for the electric field can be derived from (8) and (9) in frequency domain $[5,8]$ by eliminating the magnetic field intensity as:

$$
\nabla \times\left(\frac{1}{\mu_{r}} \nabla \times \boldsymbol{E}\right)-k_{0}^{2} \varepsilon_{r} \boldsymbol{E}=-\mathrm{j} k_{0} Z_{0} \boldsymbol{J},
$$

where $Z_{0}$ is the wave impedance of the free space,

$$
Z_{0}=\sqrt{\frac{\mu_{0}}{\varepsilon_{0}}}=120 \pi[\Omega] .
$$

On the surface of a finite space, the Sommerfeld radiation condition in frequency domain [8] can be approximated as:

$$
\boldsymbol{n} \times \nabla \times \boldsymbol{E}+\mathrm{j} k_{0} \boldsymbol{n} \times(\boldsymbol{n} \times \boldsymbol{E}) \approx 0 .
$$

\subsection{Basics of nodal finite element method}

Finite element method $[6,8-13]$ is a numerical technique developed for finding approximate solutions of differential equations. It is used to approximate analytically unsolvable or hardly solvable problems, such as electromagnetic, thermal, fluid, etc. problems. The method has four main steps [6]: 
- Specification of the model: defining the geometry, and the physics of the problem;

- Preprocessing: discretization of the geometry, finite element mesh;

- Calculation: finite element assembly, solving the equation system;

- Postprocessing: evaluation of the results.

In this paper, the preprocessing step was made in the frame of Gmsh [14,15], the calculation and the postprocessing steps were realized by Mat lab [16] functions.

To solve the approximation of the problem with the weighted residual method, the equations are multiplied by arbitrary weighting functions, but in Galerkin's method [17], the weighting functions are selected as the approximate shape functions. In this paper the nodal shape functions are used, because every example are defined with a current density perpendicular to the plane of the geometry. There are defined shape functions on each node of each element. A shape function interpolates the solution between the mesh nodes. A shape function on a node has the value of 1 , and 0 on all other nodes. In 1-dimension, the elements are straight sections. The 1-dimensional shape functions can be defined [6] as:

$$
N_{i}(x)=\prod_{j=1, j \neq i}^{m} \frac{x-x_{j}}{x_{i}-x_{j}}
$$

where $N$ is the shape function, $m$ is the number of the nodes of the element, $x_{i}$ and $x_{j}$ are the coordinates of the nodes of the element. The first and the third order shape functions can be seen on Fig. 1.
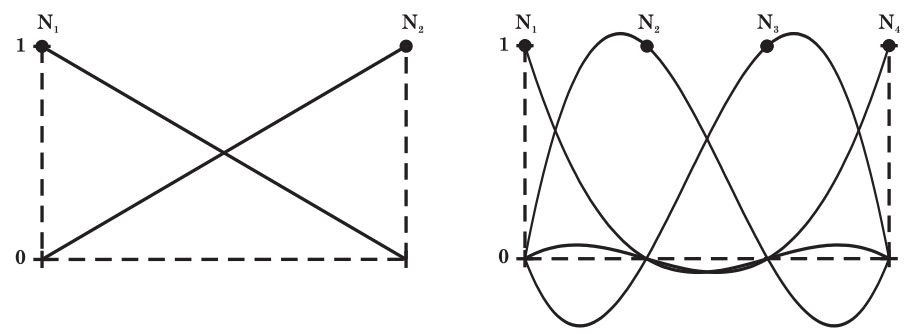

Figure 1. 1-dimensional shape functions

The 2-dimensional linear shape functions on triangle elements can be introduced [6] by using barycentric coordinates. The area of triangles can be calculated by determinants as:

$$
\Delta=\frac{1}{2}\left|\begin{array}{ccc}
1 & x_{1} & y_{1} \\
1 & x_{2} & y_{2} \\
1 & x_{3} & y_{3}
\end{array}\right|
$$


An arbitrary point in the triangle with coordinates $(x, y)$ splits it onto three different triangles (see in Figure 2). The area of the triangles can be calculated, as above, as:

$$
\Delta_{1}=\frac{1}{2}\left|\begin{array}{ccc}
1 & x & y \\
1 & x_{2} & y_{2} \\
1 & x_{3} & y_{3}
\end{array}\right|, \Delta_{2}=\frac{1}{2}\left|\begin{array}{ccc}
1 & x_{1} & y_{1} \\
1 & x & y \\
1 & x_{3} & y_{3}
\end{array}\right|, \Delta_{3}=\frac{1}{2}\left|\begin{array}{ccc}
1 & x_{1} & y_{1} \\
1 & x_{2} & y_{2} \\
1 & x & y
\end{array}\right| .
$$

Now, the 2-dimensional linear shape functions can be defined as:

$$
N_{i}=\frac{\Delta_{i}}{\Delta}
$$

The nodal finite element method can be used only, if the unknown vector space is perpendicular to the 2-dimensional geometry, or the unknown field is scalar.

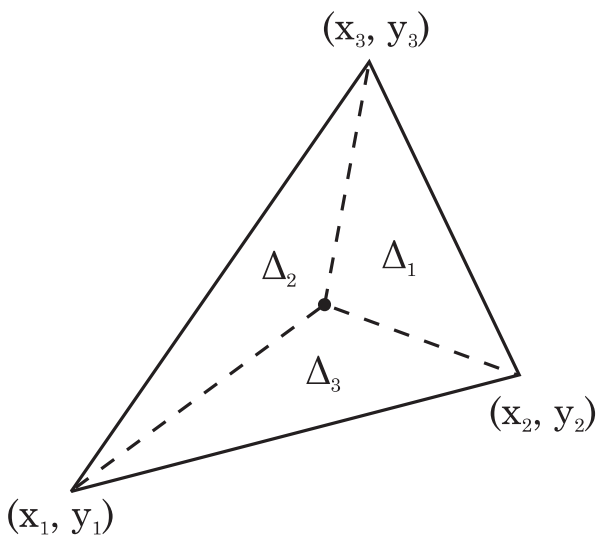

Figure 2. To understand the barycentric coordinates

\section{Weak formulations, and their implementation method}

\subsection{Equations in time domain}

To find the approximation of the solution of the time domain problem, the wave equation (14) can be multiplied with an arbitrary vector weighting function $T$ and integrated over the whole volume $V$ :

$$
\begin{aligned}
\int_{V} \boldsymbol{T} \cdot \nabla \times\left(\frac{1}{\mu} \nabla \times \boldsymbol{E}\right) \mathrm{d} V & +\int_{V} \boldsymbol{T} \cdot \varepsilon \frac{\partial^{2} \boldsymbol{E}}{\partial t^{2}} \mathrm{~d} V+\int_{V} \boldsymbol{T} \cdot \sigma \frac{\partial \boldsymbol{E}}{\partial t} \mathrm{~d} V= \\
& -\int \boldsymbol{T} \cdot \frac{\partial \boldsymbol{J}}{\partial t} \mathrm{~d} V .
\end{aligned}
$$


This equation is the so called direct formulation, that contains second order derivatives. It can be eliminated by invoking the vector algebraic identity [9]:

$$
\nabla \cdot(\boldsymbol{x} \times \boldsymbol{y})=\boldsymbol{y} \cdot \nabla \times \boldsymbol{x}-\boldsymbol{x} \cdot \nabla \times \boldsymbol{y},
$$

with $\boldsymbol{x}=\boldsymbol{T}$ and $\boldsymbol{y}=\mu^{-1} \nabla \times \boldsymbol{E}$, and the approximation of the Sommerfeld radiation condition (15), the weak form of the time domain analysis [8] can be represented as:

$$
\begin{gathered}
\int_{V}(\nabla \times \boldsymbol{T}) \cdot \frac{1}{\mu}(\nabla \times \boldsymbol{E}) \mathrm{d} V+\int_{V} \boldsymbol{T} \varepsilon \frac{\partial^{2} \boldsymbol{E}}{\partial t^{2}} \mathrm{~d} V+\int_{V} \boldsymbol{T} \sigma \frac{\partial \boldsymbol{E}}{\partial t} \mathrm{~d} V \\
+Y_{0} \oint_{A}(\boldsymbol{n} \times \boldsymbol{T}) \cdot\left(\boldsymbol{n} \times \frac{\partial \boldsymbol{E}}{\partial t}\right) \mathrm{d} A=-\int \boldsymbol{T} \cdot \frac{\partial \boldsymbol{J}}{\partial t} \mathrm{~d} V
\end{gathered}
$$

where $S$ is the integration variable on surface. The finite element method use the weak formulation with Galerkin's-method when the weighting function and the shape function are the same. The solution of the electric field within each element using the shape functions can be expressed $[6,8]$ as:

$$
E(r)=\sum_{i=1}^{m} N_{i}(r) E_{i} .
$$

Rewriting (27) into (26) results in a second order, inhomogeneous differential equation system [8]:

$$
\mathbf{Q} \frac{\partial^{2} \mathbf{e}}{\partial t^{2}}+\mathbf{R} \frac{\partial \mathbf{e}}{\partial t}+\mathbf{S} \mathbf{e}=\mathbf{f}
$$

where:

$$
\begin{gathered}
Q_{i, j}=\int_{V} \boldsymbol{T}_{i} \cdot \varepsilon \boldsymbol{T}_{j} \mathrm{~d} V \\
R_{i, j}=\int_{V} \boldsymbol{T}_{i} \cdot \sigma \boldsymbol{T}_{j} \mathrm{~d} V+Y_{0} \oint_{A}\left(\boldsymbol{n} \times \boldsymbol{T}_{i}\right) \cdot\left(\boldsymbol{n} \times \boldsymbol{T}_{j}\right) \mathrm{d} A, \\
S_{i, j}=\int_{V}\left(\nabla \times \boldsymbol{T}_{i}\right) \frac{1}{\mu}\left(\nabla \times \boldsymbol{T}_{j}\right) \mathrm{d} V, \\
f_{i}=-\int_{V} \boldsymbol{T}_{i} \cdot \frac{\partial \boldsymbol{J}}{\partial t} \mathrm{~d} V,
\end{gathered}
$$

and $\mathbf{e}$ is the columnvector of the unknown electric field. An unconditionally stable method to solve numerically a second order differential equation is the so called Newmark- $\beta$ method [19]. Using the central difference form for the first and the second order time derivatives:

$$
\begin{gathered}
\frac{\partial u(t)}{\partial t} \approx \frac{u(t)^{n+1}-u(t)^{n-1}}{2 \Delta t}, \\
\frac{\partial^{2} u(t)}{\partial t^{2}} \approx \frac{u(t)^{n+1}-2 u(t)^{n}+u(t)^{n-1}}{\Delta t^{2}}
\end{gathered}
$$


and the weighted average for undifferentiated quantities:

$$
\begin{gathered}
\mathbf{e} \approx \beta \mathbf{e}^{n+1}+(1-2 \beta) \mathbf{e}^{n}+\beta \mathbf{e}^{n-1}, \\
\mathbf{f} \approx \beta \mathbf{f}^{n+1}+(1-2 \beta) \mathbf{f}^{n}+\beta \mathbf{f}^{n-1},
\end{gathered}
$$

where $\beta$ is a parameter between 0 and 1 , substituting these into (28), the form of the time-stepping equation system is:

$$
\begin{gathered}
\left(\mathbf{Q} \frac{1}{\Delta t^{2}}+\mathbf{R} \frac{1}{2 \Delta t}+\beta \mathbf{S}\right) \mathbf{e}^{n+1}=\left(\frac{2}{\Delta t^{2}} \mathbf{Q}-(1-2 \beta) \mathbf{S}\right) \mathbf{e}^{n} \\
-\left(\mathbf{Q} \frac{1}{\Delta t^{2}}-\mathbf{R} \frac{1}{2 \Delta t}+\beta \mathbf{S}\right) \mathbf{e}^{n-1}+\beta \mathbf{f}^{n+1}+(1-2 \beta) \mathbf{f}^{n}+\beta \mathbf{f}^{n-1} .
\end{gathered}
$$

It is easy to see, that (37) is now a solvable equation system, where $\mathbf{e}^{n+1}$ is the unknown vector, and the right hand side of the equation is known from the time steps earlier. This method is unconditionally stable, if $\beta \geq \frac{1}{4}$ [8].

\subsection{Equations in frequency domain}

Multiplied the wave equation in frequency domain (17) with an arbitrary vector weighting function $\boldsymbol{T}$ and integrated over the whole volume $V$ :

$$
\int_{V} \boldsymbol{T} \cdot\left(\nabla \times \frac{1}{\mu_{r}} \times \boldsymbol{E}\right) \mathrm{d} V-\int_{V} \boldsymbol{T} \cdot k_{0}^{2} \epsilon_{r} \boldsymbol{E} \mathrm{d} V=-\int_{V} \boldsymbol{T} \cdot \mathrm{j} \omega \mu_{0} \boldsymbol{J} \mathrm{d} V .
$$

This equation contains second order derivatives. It can be eliminated by using the vector algebraic identity in (25) with $\boldsymbol{x}=\boldsymbol{T}$ and $\boldsymbol{y}=\mu_{r}^{-1} \nabla \times \boldsymbol{E}$, then the Gauss-Ostrogradskij theorem [6]:

$$
\oint_{A} \boldsymbol{v} \cdot \mathrm{d} \boldsymbol{A}=\int_{V} \nabla \cdot \boldsymbol{v} \mathrm{d} V
$$

with $\boldsymbol{v}=\boldsymbol{T} \times\left(\mu_{r}^{-1} \nabla \times \boldsymbol{E}\right)$ and the approximation of the Sommerfeld radiation condition(19), the weak formulation of the frequency domain analysis is:

$$
\begin{gathered}
\int_{V}(\nabla \times \boldsymbol{T}) \cdot \frac{1}{\mu_{r}}(\nabla \times \boldsymbol{E}) \mathrm{d} V-\int_{V} \boldsymbol{T} \cdot k_{0}^{2} \epsilon_{r} \boldsymbol{E} \mathrm{d} V \\
+\mathrm{j} k_{0} \oint_{A}(\boldsymbol{n} \times \boldsymbol{T}) \cdot(\nabla \times \boldsymbol{E}) \mathrm{d} A=\int_{V} \boldsymbol{T} \cdot \mathrm{j} k_{0} Z_{0} \boldsymbol{J} \mathrm{d} V,
\end{gathered}
$$

Substituting (27) into the weak form of (40), the result is a simple equation system in the form:

$$
\mathbf{K e}=\mathbf{b},
$$


where

$$
\begin{gathered}
K_{i, j}=\int_{V}\left[\left(\nabla \times \boldsymbol{T}_{i}\right) \cdot \frac{1}{\mu_{r}}\left(\nabla \times \boldsymbol{T}_{j}\right)-k_{0}^{2} \boldsymbol{T}_{i} \cdot \epsilon_{r} \boldsymbol{T}_{j}\right] \mathrm{d} V+ \\
\mathrm{j} k_{0} \oint_{A}\left(\boldsymbol{n} \times \boldsymbol{T}_{i}\right) \cdot\left(\boldsymbol{n} \times \boldsymbol{T}_{j}\right) \mathrm{d} A,
\end{gathered}
$$

and

$$
b_{i}=\int_{V} \boldsymbol{T}_{i} \cdot \mathrm{j} k_{0} Z_{0} \boldsymbol{J} \mathrm{d} V .
$$

The simulations can be evaluated for only one frequency at one case. Along the boundaries, which are not in vacuum/air, the wave number of the surface integral in (42) should be the wave number calculated from the parameters of the boundary material.

\section{Examples}

The following simulations will show the advantages and disadvantages of the time and frequency domain simulations. If it is possible, the accuracy of the models will be shown compared to analytical results.

\subsection{Simulations in time domain}

\subsubsection{Perfect electrically conducting wall}

A perfect electrically conducting wall reflects the electromagnetic wave with a reflexion coefficient -1 . The first simulated geometry is a 3 meter long line, an excitation is placed at 1 meter, and the boundary on the right side is a perfectly conducting wall. The conducting wall is implemented as a simple Dirichlet boundary, where the electric field is set to be zero. A Dirichlet-type boundary condition defines the exact value of the solution. The left boundary must be defined as an absorbing boundary to grant the wave propagation without reflexion on the wall. This is a Neumann type boundary condition, which means, during the assembly, to the value $R_{i, i}$ (See in (30)), where $i$ is the number of the node, one has to add the value of $Y_{0}$. A Neumann-type boundary condition specifies the derivative of the solution on a boundary. Two simulations were made on one frequency and one simulation was made with a combined signal (See in Figure 3). The results in Fig. 3 are the maximal values on each element after the transient. If the applied frequency is $150 \mathrm{MHz}$, the distance between the source and the wall is the half of the wavelength, so the sum of the incident and the reflected wave is zero, but at $225 \mathrm{MHz}$, the distance is three-quarter of the wavelength, so the incident wave meets the reflected wave at the antenna in equal phase. 


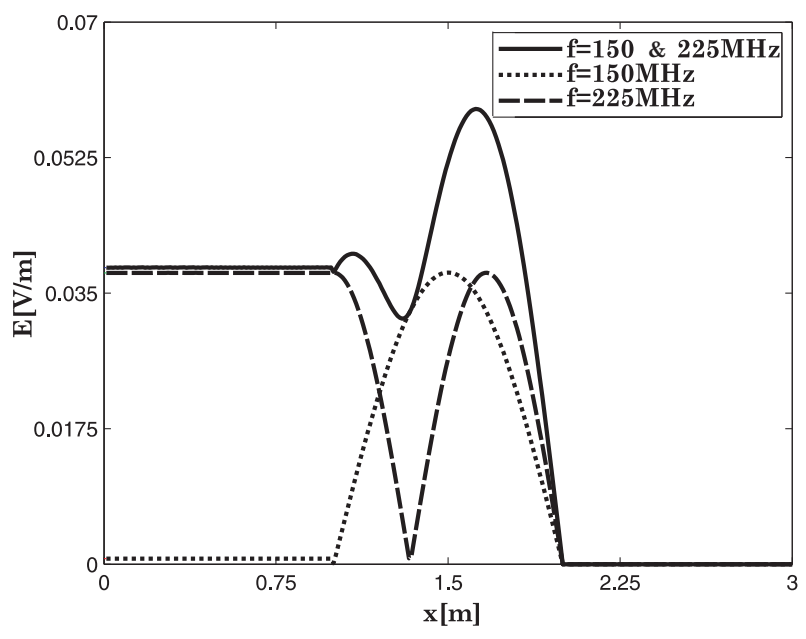

Figure 3. Results with perfect electrically conducting wall

\subsubsection{Reflexion on the boundary of two different media}

On the boundary of two media, if the wave impedances differ from each other, reflexion occurs, what can be calculated as:

$$
\Gamma=\frac{Z_{02}-Z_{01}}{Z_{02}+Z_{01}},
$$

where $\Gamma$ is the reflexion coefficient, $Z_{02}$ and $Z_{01}$ are the wave impedance of the two media. The geometry is a 5 meter long line, which is separated into two part (See in Figure 4). In time domain, the reflexion can be calculated from the incident and the reflected wave,

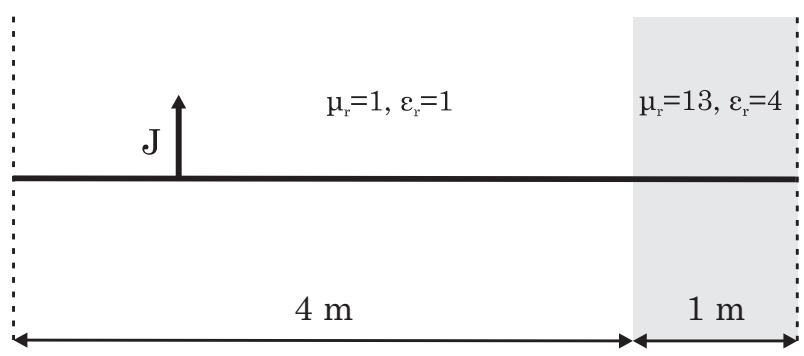

Figure 4. Applied geometry for the reflexion simulation

or from the standing wave ratio. The left and the right boundary are both absorbing boundaries, but on the right side it has different wave admittance. The result can be seen 


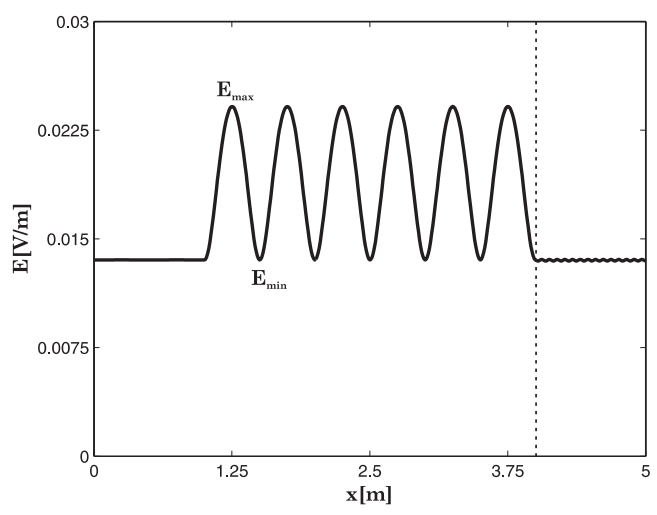

Figure 5. Results with two different media

on Fig. 5. The maximal and the minimal value of the electric field can be read from the results. The calculated reflexion coefficient is 0.286 , and the simulated is 0.285 .

\subsubsection{Rectangular cavity rezonator}

Analyzing resonators and their quality factors requires time domain simulations, because transients can be analyzed only in the time domain. The transient analysis is needed, because the oscillation is the transient response of the resonators. A resonator with perfectly conducting walls, used on the resonance frequency, has an infinite quality factor. The geometry of the applied problem is a simple square, witch size of the sides are 0.1 meter (See in Figure 6). The resonance frequency of this resonator of the mode $T E_{101}$ [5] is $1.0606 \mathrm{GHz}$. The excitation is one period at the resonance frequency. Figure 7 shows the results of an ideal rectangle cavity and a rectangle cavity with badly conducting walls. The boundary of the rectangle cavity with the loss has the properties $\sigma=10[\mathrm{~S} / \mathrm{m}]$, and $\mu_{r}=\varepsilon_{r}=2$. The excitation is perpendicular to the plane of the geometry. The wall of

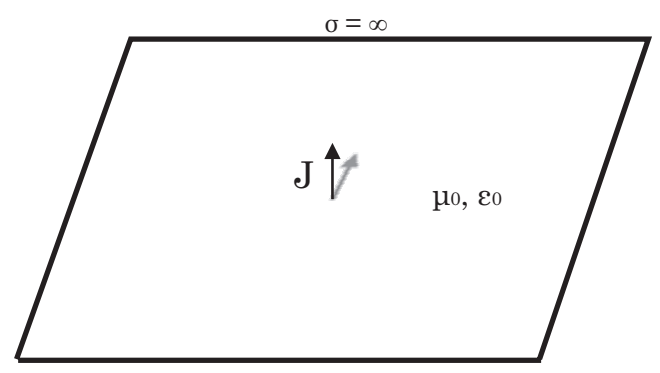

Figure 6. Model of the rectangular cavity 
the resonator is set as a perfect electrically conducting surface, the electric field must be perpendicular to the perfectly conducting surface. In the 2-dimensional simulations, where the excitation is perpendicular to the simulation plane, the electric field is parallel to the source current, so the electric field is zero on the conducting wall in this case. In Fig. 7, the dotted line shows the case with perfeclty conducting walls, and the continuous line is for the electric field in the cavity with badly conducting walls. The quality factor of the

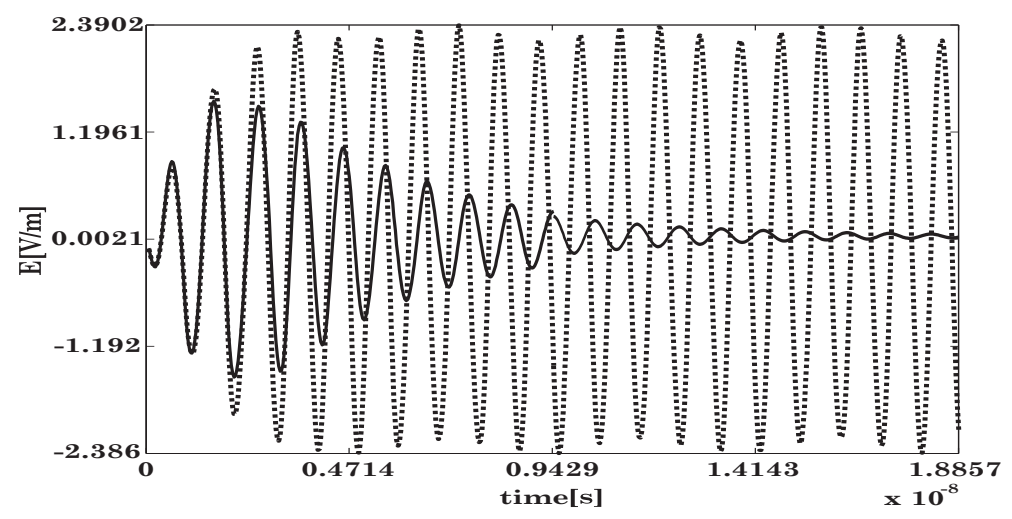

Figure 7. Electric field in the center of rectangular cavity resonator

rectangular cavity can be defined as the number of period till the amplitude of the electric field reaches the value of the original amplitude divided by the Euler number $e$. Here the value of quality factor is approximately about 6 .

Time domain simulations are very time consuming, because every time step must be calculated. The transient analysis is only possible in time domain, but for the most of the high frequency problems, it is not needed to calculate the transient behavior.

\subsection{Simulations in frequency domain}

\subsubsection{Perfect electrically conducting wall}

The following problem, the geometry and the excitation is the same as in the section 3.1.1.The method of implementing of the conducting and absorbing wall is the same as in time domain. The result of a frequency domain simulation is a complex value. The absolute value and the phase of the result can be seen on Fig. 8 and Fig. 9. The figures above show the result without the conducting wall. The absolute values of the results are the same as in time domain. The phase is constant between the excitation and the wall, which means, that standing waves are formed there. The linear parts of the phase plot mean simple wave propagating. 

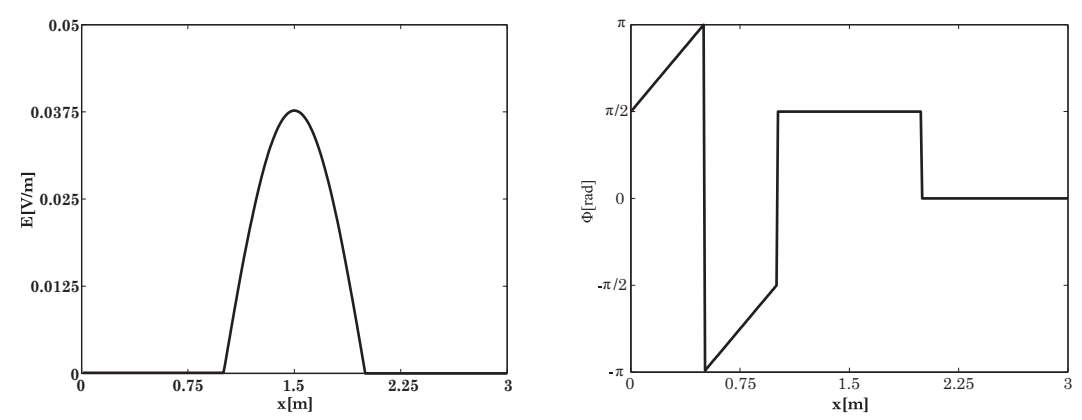

Figure 8. The absolute value and the phase of the result at $150 \mathrm{MHz}$
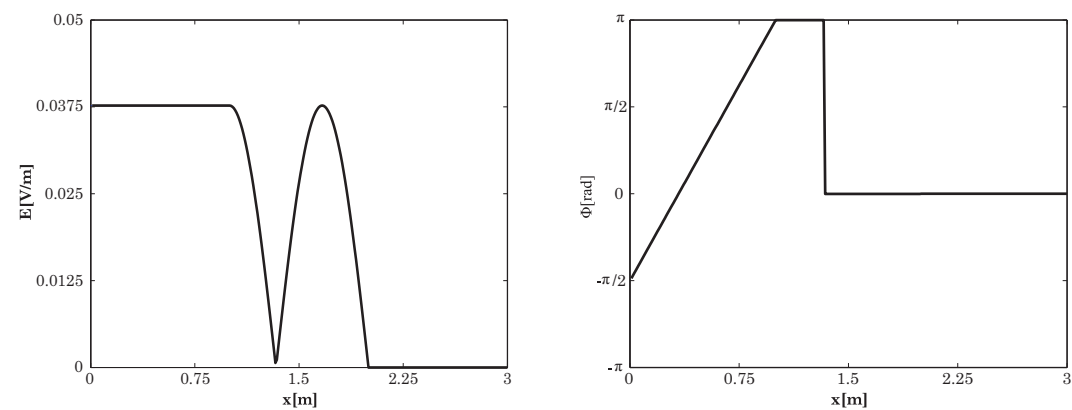

Figure 9. The absolute value and the phase of the result at $225 \mathrm{MHz}$

\subsubsection{Rectangular waveguide}

This simulation is a about a simple rectangular waveguide in $T E_{10}$ mode. The model of the geometry can be seen in Fig. 10. The width of the waveguide is $0.1 \mathrm{~m}$, so the

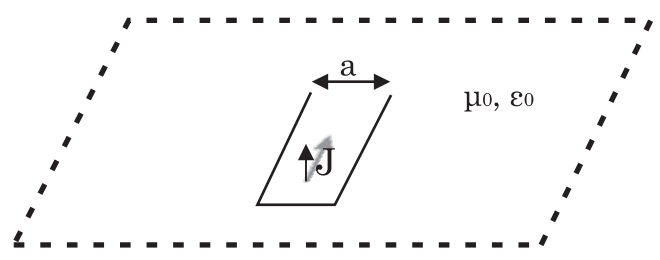

Figure 10. The model of the simulated rectangular waveguide

cutoff frequency of this arrangement is $1.5 \mathrm{GHz}$. The frequency of the excitation is chosen as $2 \mathrm{GHz}$, from the value of the cutoff frequency and the applied frequency the inguide wavelength van be calculated, witch is $0.2268 \mathrm{~m}$. Fig. 11 shows the absolute value and the phase of the result of the simulation. On Fig. 11 it can be seen that the open ended 

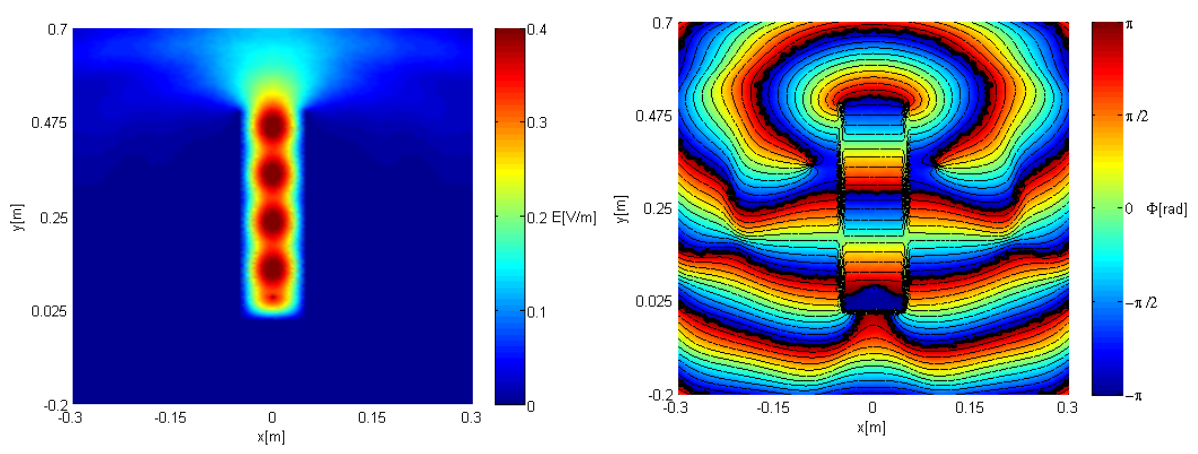

Figure 11. The absolute value and the phase of the result

rectangular waveguide radiates backward. The guide wavelength can be measured from the phase plot, in this case it is approximately $0.228 \mathrm{~m}$. The error is in the order of magnitude of the size of the elements in the waveguide.

\subsubsection{Parabolic antenna}

Parabolic antennas are widely used by satellite communication systems owing to their polar pattern. Due to the special geometry of the parabolic antennas, plane waves are propagating parallel to their axis. The geometry and the mesh are shown on Fig. 12. The

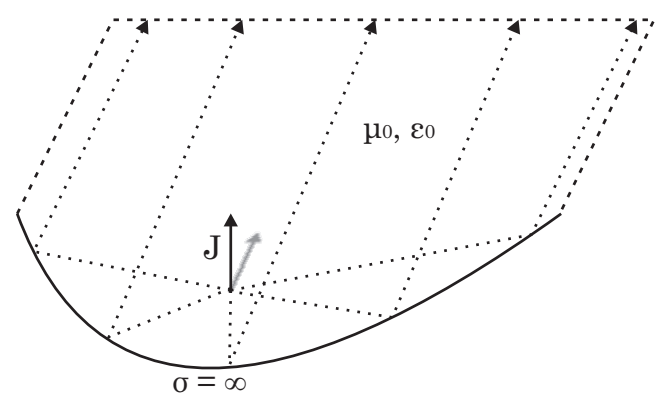

Figure 12. The model of the simulated parabolic antenna

frequency of the simulation was $300 \mathrm{MHz}$. In practice, the size of the mirror is much larger, than the wavelength of the signal, the simulation was made on lower frequency for the better visibility, and to decrease the number of unknowns. The mirror is set as a perfect electrically conducting surface. Fig. 13 shows the absolute value and the phase of the result. It can be seen on the plot of the phase, that the wave propagates parallel to the axis of the mirror as an almost perfect plane wave. The real parabolic antennas work on higher frequencies, and have a better polar pattern. 

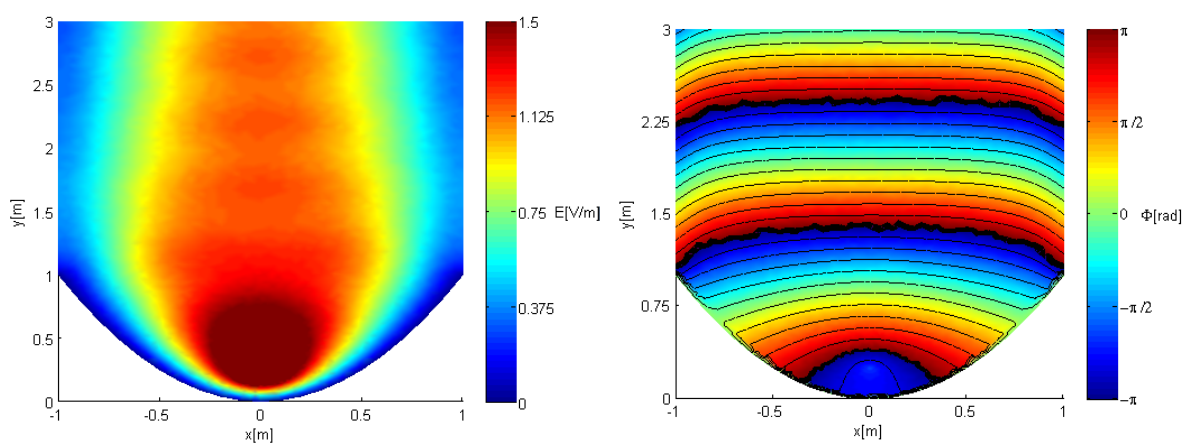

Figure 13. The absolute value and the phase of the result

The simulations in frequency domain give fast result for the problems, but only at one frequency at a time. In practice, the most important question is the behavior in, and out of the applied frequency range of any device.

\section{Conclusions}

In this paper, simple 1-D and 2-D electromagnetic field problems were presented in time and frequency domain. The first chapter is a short theoretical summary of the wave equations expressed to the electric field and of the nodal finite element method as well. In the second chapter the deriving of the applied weak forms in time and frequency domain and their application methods were discussed. These methods were presented via some simple examples. The simulations represent the physical behavior well. Time domain simulation is very effective to find the result with an appropriate input signal and to calculate the transients phenomena, however, it can be very time consuming, because the field must be calculated in every time step. In contrast, a frequency domain simulation can be done only at one frequency at a time, but the implementation of it is much easier, it gives a fast result, and in practice, the behavior of the devices in frequency domain is much more important. In the future, the edge elements will be implemented to simulate complex problems, where the excitation is in the plane of the simulation.

\section{Acknowledgement}

This research was supported by the European Union and the State of Hungary, cofinanced by the European Social Fund in the framework of TÁMOP-4.2.4.A/ 2-11/12012-0001 'National Excellence Program'. 


\section{References}

[1] Maxwell, J. C.: A Dynamical Theory of the Electromagnetic Field, Philosophical Transactions of the Royal Society, London, 1865

[2] Simonyi, K., Zombory, L.: Theoretycal Electricity (in Hungarian), Múszaki Könyvkiadó, Budapest, 2000

[3] Fodor, Gy.: Electromagnetic Fields (in Hungarian), Múegyetemi Kiadó, Budapest, 1996

[4] Standeisky, I.: Electrodynamics (in Hungarian), Universitas, Győr, 2006

[5] Pozar, D. M.: Microwave Engineering, John Wiley and Sons, New York, 2012

[6] Kuczmann, M., Iványi, A.: The Finite Element Method in Magnetics, Academic Press, Budapest, 2008

[7] Jackson, J. D.: Classical Electrodynamics, John Wiley and Sons, New York, 1999

[8] Jin, J.: Finite Element Analysis of Antennas and Arrays, John Wiley and Sons, New York, 2009

[9] Jin, J.: The Finite Element Method in Electromagnetics, John Wiley and Sons, New York, 2002

[10] Gustrau, F., Manteuffel, D.: EM Modeling of Antennas and RF Components for Wireless Communication Systems, Springer, Berlin, 2006

[11] Pepper, D. W., Heinrich, J. C.: The Finite Element Method, Taylor and Francis, New York, 2006

[12] Kuczmann, M.: Vector Preisach Hysteresis Modeling: Measurement, Identification and Application, Physica B - Condensed Matter, vol. 406, no. 8, pp. 1403-1409, 2011 DOI: 10.1016/j.physb.2011.01.037

[13] Kuczmann, M., Budai, T., Kovács, G., Marcsa, D., Friedl, G., Prukner, P., Unger, T., Tomozi, Gy.: Application of PETSC and other useful packages in finite element simulation, Pollack Periodica, vol. 8, no. 2, pp. 141-148, 2013

DOI: 10.1556/Pollack.8.2013.2.15

[14] Gmsh, http://geuz.org/gmsh/, (last visited: 05 February 2014)

[15] Geuzaine, C., Remacle, J. F.: Gmsh: a three-dimensional finite element mesh generator with built-in pre- and post-processing facilities, International Journal for Numerical Methods in Engineering, vol. 79, no. 11, pp. 1309-1331, 2009

DOI: $10.1002 / n m e .2579$ 
[16] Matlab, www.mathworks.com, (last visited: 05 February 2014)

[17] Iványi, A.: Continous and Discrete Simulations in Electrodynamics (in Hungarian), Akadémiai Kiadó, Budapest, 2003

[18] Bronshtein, I. N., Semendyayev, K. A., Musiol, G., Muehling, H.: Handbook of Mathematics. Typotex, Budapest, 2009

[19] Newmark, N. M. A method of computation for structural dynamics, Journal of Engineering Mechanics, ASCE, 1959 